\title{
HST Eclipse Studies of Magnetic CVs: Resolving the UV Hot Spot and Accretion Stream
}

\author{
H.S. Stockman \\ Space Telescope Science Institute ${ }^{1}, 3700$ San Martin Drive, Baltimore, \\ $M D 21218$
}

\begin{abstract}
Time-resolved photometry and spectroscopy of cataclysmic variables $(\mathrm{CV})$ are powerful tools for clarifying the physical size, placement, and conditions of emission regions in these compact systems. We present HST time resolved UV spectroscopic data for one self-eclipsing mCV system, ST LMi, and two secondary eclipsing systems, DP Leo and UZ For. These observations clearly show the relative contributions of three emission components: the white dwarf, a hotter region surrounding the accretion shock, and the photoionized accretion stream. UZ For was observed in an unusually active state by both HST and EUVE. These data provide strong evidence that the accretion stream is "clumpy", with overdensities 100-1000 times the average density. Using photoionization codes such as XSTAR and CLOUDY, we find that steady, supersonic, and homogenous flow in the UZ For accretion stream is unstable to compression heating. As a consequence, we propose a two-phase model for the stream and obtain density enhancements comparable to those deduced from the strengths of the UV emission lines and EUVE absorption features. Similar behavior may be expected from active, asynchronous magnetic CVs with coupling radii greater than several white dwarf radii.
\end{abstract}

\section{Introduction}

The Magnetic Cataclysmic Variables (mCVs) or "Polars", like non- magnetic $\mathrm{CVs}$, are short period binaries with a low-mass companion in contact with a degenerate white dwarf. Roche lobe overflow, driven by magnetic braking of the secondary or gravitational radiation, provides a flow of material from the surface of the secondary star to the white dwarf. In the synchronous mCVs, the magnetic field from the primary star halts and threads the material well away for the white dwarf surface, $R \gtrsim 10 R_{1}$, and prevents an accretion disk from forming. The threaded material is channeled to the surface where the kinetic energy of infall is converted into heat in a relatively small and localized accretion shock(s). The first self-consistent models of these systems (e.g. Lamb \& Masters 1978) could explain many of the observable features of these systems: the hard $\mathrm{X}$-ray spectrum due to bremsstrahlung cooling at the surface; the highly polar-

\footnotetext{
${ }^{1}$ Operated by the Association of Universities for Research in Astronomy, Inc. for the National Aeronautics and Space Administration
} 
ized optical cyclotron emission which is the hallmark of these systems; and the strong soft X-ray flux $\left(k T_{s x} \gtrsim 25 \mathrm{eV}\right)$ which arises from reprocessing of the Xray radiation by the surrounding photosphere. However, simple, homogeneous shock models fail to predict the correct balance between the soft $\mathrm{X}$-ray luminosity and the primary coolants (cyclotron and bremsstrahlung, Beuermann 1988, Ramsay et al. 1994 ). Nor does a single region for the soft X-ray, hard X-ray, and cyclotron emission appear consistent with eclipse data and other physical constraints ( Stockman 1995). Most models which have been proposed to explain these discrepancies involve an inhomogeneous accretion flow, in which the soft X-ray flux arises from the regions of high density flow in which the shock surface is actually submerged below the surface of the white dwarf and much of the optical cyclotron is created in a low- density "halo" surrounding the soft X-ray region(s) (Kuijpers \& Pringle 1982, Frank, King, \& Lasota, J.-P. 1988). In this "halo", the accretion rate is sufficiently low $\left(\dot{m} \lesssim 1 \mathrm{gm} / \mathrm{cm}^{2}-s\right)$ and the cyclotron cooling so great that infalling nucleons interact directly with the underlying photosphere rather than make a strong, adiabatic shock. These models are capable of explaining the observed cyclotron spectrum in several sources(e.g. Woelk \& Beuermann 1993, Beuermann, et al 1996). They are unable to explain, however, the origin or geometry of the "blobby" accretion flow and any dependence it may have on the overall accretion rate or magnetic field strength. The HST UV eclipse studies are motivated by these issues and how the origin and site of the hot UV continuum may contribute to our understanding of these interesting systems.

\section{HST UV Eclipse Data}

We have obtained time-resolved data for three mCVs. As is often the case, each system presented a different behavior, either due to activity level or the eclipsing geometry. We summarize the results of these studies below:

\subsection{DP Leo}

We observed DP Leo in a low activity state. Only weak C IV $\lambda 1549$ is detected in the UV spectrum. However, the system is undoubtedly active, with evidence of a hot, blue continuum with $T_{U V} \gtrsim 30,000 \mathrm{~K}$. The hot spot appears to be resolved by the eclipse data and we obtain an "radial" extent of $\sim 0.15 R_{1}$. These data are consistent with the optical and hard X-ray light curves of Bailey \& Cropper (1991), However, the size and temperature of the hot spot cannot be extrapolated to the soft X- ray luminosity observed by Robinson \& Cordova (1994). Thus, the UV hot spot is best attributable to the reprocessing under a low density accretion "halo" with a temperature, fractional coverage in the range $T_{5} f=0.005-0.009$. Here, $T_{5}$ is the temperature of the hot spot in units of $100,000 \mathrm{~K}$ and $f$ is the fractional area of the white dwarf covered by the hotspot. By contrast, typical soft X-ray regions have $T_{5} f \sim 0.001$.

\subsection{ST LMi}

ST LMi was observed prior to and during self-eclipse egress. During the observation, the primary shock rotates over the limb of the white dwarf and into our view. In the UV, we observe a slow but significant increase in the far UV band 
during the egress - consistent with the appearance of a large optically thick spot (e.g. Cropper \& Horne 1994)- and very little increase in the near-UV light. Clearly the accretion column and white dwarf photospheric emission dominates the near UV. We can estimate the temperature of the hot spot by differencing the pre-egress and post-egress spectra and obtain $T_{5} f=0.002-0.008$.

\subsection{UZ For}

The most interesting observations were two eclipses of UZ For during an active state (Stockman \& Schmidt 1996). We can resolve the eclipse of the white dwarf, the UV hot spot, and the extended eclipse of the UV-bright accretion column. The temperature, fractional coverage of the UV hot spot was quite large, approximately $T_{5} f=0.02-0.06$ and is inconsistent with an extrapolation of the soft X-ray region's size and flux. The spectrum of the UV hot spot, which we obtained from decomposing the eclipse light curves into separate wavelength regions, was not well fit by a single temperature and may be due to a wide range of accretion flows (e.g. as in AM Her) or due to heating of the photosphere by "reflected" continuum from the base of the accretion column.

The eclipse of the emission lines and the near-UV portion of the spectrum indicate that the accretion stream extends to $R \sim 12 R_{1}$ from the surface and further than the expected bend in the ballistic stream of material flowing from the secondary. Moreover, the line emission eclipse ingress appears before white dwarf ingress, indicating that much of the line emission originates from material which is threaded very close to the $L_{1}$ Roche lobe.

\section{Modeling the Accretion Stream}

To further interpret the UV spectra, we used the photoionization codes XSTAR and CLOUDY to predict the ionization balance and radiation from the accretion column. The column was divided into 8 logarithmic radial sections and the density and velocity shear were input using the observed EUVE luminosity (estimated to be $L_{s x}=1.410^{33} \mathrm{ergs} / \mathrm{s}$ ) and the system parameters of Bailey \& Cropper (1991). A soft X-ray temperature of $k T_{s x}=25 \mathrm{eV}$ and a shock covering factor of $f_{\text {shock }}=0.01$ were assumed. We studied the conditions of the accretion column for 5 different "filling factors", $\epsilon=\bar{\rho}^{2} / \bar{\rho}^{2}=1 ., 0.1,0.01,0.001$ and a $10 \%$ underdense but uniformly filling accretion stream. The results of the models are shown in figure 1.

We found that a free-falling underdense stream is unstable to shocks and compression heating, with $T_{\text {gas }} \rightarrow T_{\text {virial }}$. Such a stream would stagnate and only begin to freefall toward the surface at a radius where cyclotron cooling becomes efficient (not included in these models).

For $\epsilon=0.1-1.0$, the stream is highly ionized and optically thin at the $\mathrm{He}$ II edge. Line and continuum emission is negligible.

For $\epsilon=0,01$, the Balmer lines are optically thin, but the UV and Balmer continuum emission increases sharply as the stream becomes optically thick to radiation above $54 \mathrm{eV}$.

For $\epsilon=0.001$, the entire column is optically thick at He II $(54 \mathrm{eV})$. Thus, the upper portion of the column, which covers a large fraction of the sky as seen from the white dwarf, can efficiently reprocess the soft $X$-rays from the base of 

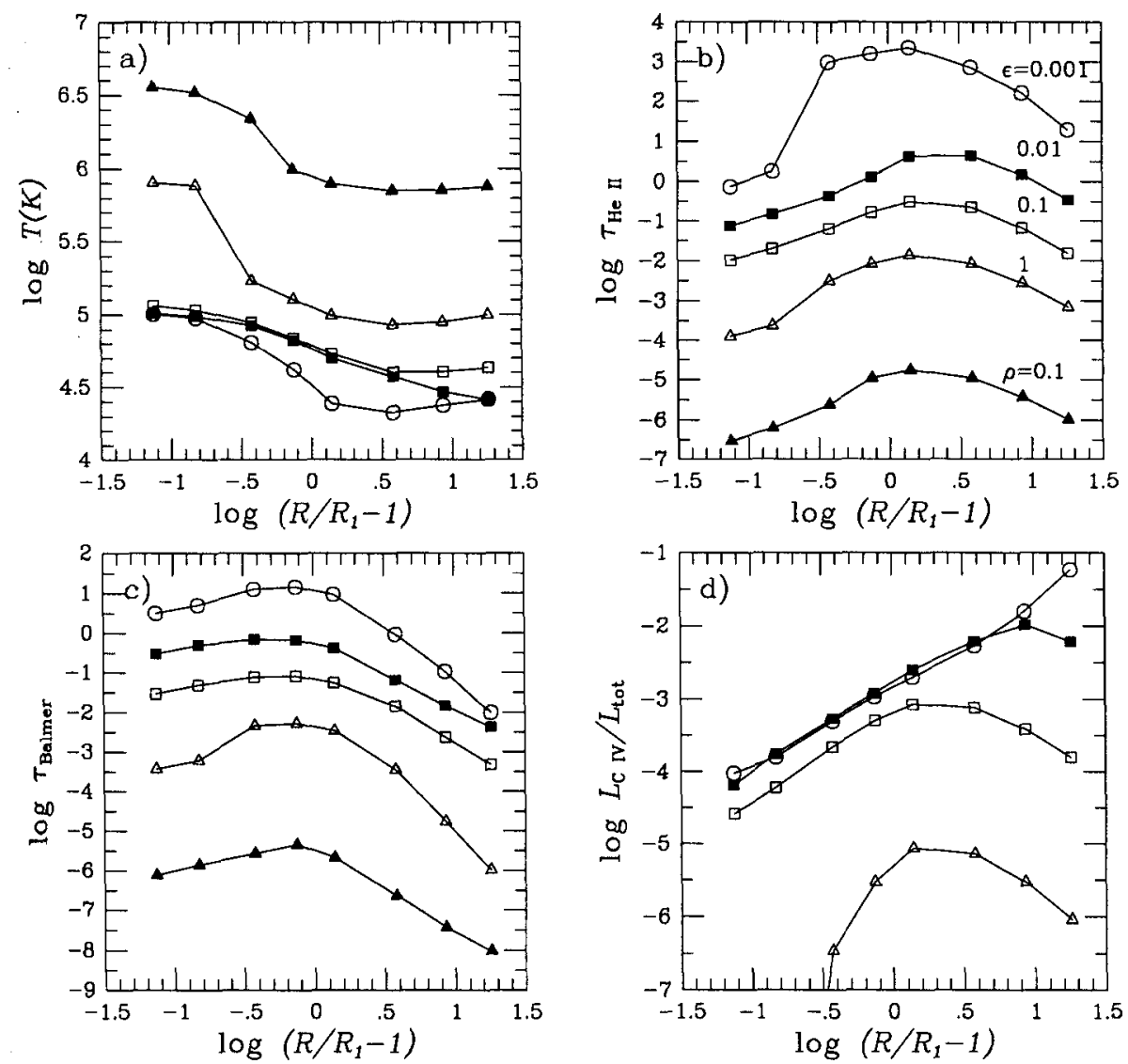

Figure 1. UZ For Accretion Column Model: Panels a)-d) indicate the average temperature, He II ( $54 \mathrm{eV})$ optical depth, Balmer continuum optical depth, and the C IV $\lambda 1549$ emission $/ L_{\text {tot }}$ versus radius for five filling factors: $\epsilon=1(0.1 \bar{\rho})$ (closed triangles), 1 (open triangles), 0.1 (open squares), 0.01 (closed squares) and 0.001 (open circles). (Stockman \& Schmidt 1996) 
the column. Since the UZ For luminosity in the Balmer continuum and C IV $\lambda$ 1549 line is $3.8 \% \& 0.6 \% L_{\text {tot }}$ respectively, a column with $\epsilon=0.01-0.001$ is most consistent with these findings. Similar flux ratios were observed in ST LMi. We note that a more collimated column, with $f \sim 0.001$ cannot intercept sufficient soft X-ray luminosity unless the column widens more rapidly than a dipole.

The "clumpy" accretion flow is consistent with the notion of dense "blobs" penetrating the photosphere, where the "blobs" uniformly fill the accretion column - unlike a "core-halo" accretion model. We also note that a "clumpy" accretion stream can have significant opacity at the He II (54 eV) edge even directly above the soft $\mathrm{X}$-ray region. This opacity may be responsible for the broad dips observed in the EUVE data for UZ For and the variations in the low energy cuttoff observed in ASCA hard X-ray spectra of UZ For. Although more detailed modeling should be done to verify this result, we expect that self-absorption by the lower part of the accretion column is important in active systems and may explain the differential heating of the secondary star observed in these systems. In addition, the interpretation of absorption features in the soft $\mathrm{X}$-ray spectra of $\mathrm{mCVs}$ should consider the effects of an ionized, but relatively cool absorbing column above the soft X-ray hot spot (e.g. Paerels et al 1996)

\section{A Two-Phase Accretion Column}

There are several reasons to consider the possibility of a two-phase accretion column, in which dense "clumps" free-fall through a subsonic, low density medium. First, "clumps" torn from the accretion stream will expand more rapidly than they can be threaded. Thus, it is likely that a hot, low density region will be created and may even dominate the upper portions of the accretion column. Second, the ratio of the equilibrium temperatures for underdense gas and dense "clumps" with $\epsilon=0.001$ is approximately the same as the density contrast, suggesting that rough pressure equilibrium is attained. Finally, a stagnant pool of hot gas would enhance the interaction between the ballistic accretion stream and the magnetic field by lowering the Alfven velocity and Kelvin-Helmholtz growth times.

It should be possible to observe the effects of a low density, hot column through its cooling processes. Bremsstrahlung and Compton cooling of the hot phase gas haave cooling times orders of magnitude longer than the dynamical time scales (by definition). However thermal conduction between the two gas phases appears to be a viable method for cooling the hot phase gas. The presence of strong thermal conduction may be discernible by analysis of UV spectra arising from the heated cool "clumps". A second coolant is cyclotron radiation. High in the column the peak wavelength for cyclotron radiation can be estimated using the approximations of Dulk \& Marsh (1982): $\lambda_{\text {peak }}(R) \sim 10 \mu \mathrm{m}\left(B_{30}\right)^{-0.9}\left(R / 2.4 R_{1}\right)^{2.3}$ with weak dependence on the accretion rate - assumed normal in this example - and white dwarf mass. Here $B_{30}$ is the polar field strength in units of 30 megagauss. The observed $10 \mu \mathrm{m}$ flux is approximately $F_{10} \sim 8(f / 0.01) D_{100}^{-2} B_{30}^{0.5}$ mJy where $D_{100}$ is the distance in units of 100 parsecs. We have chosen $10 \mu \mathrm{m}$ as a fiducial wavelength since AM Her was detected at $10 \mu \mathrm{m}$ at a level roughly consistent with these predictions (Stockman et al 1977). The observed cyclotron peak from the hot column indicates the radius 
at which the hot column rapidly cools and free-falls to the surface. The peak wavelength depends most strongly on the hot phase accretion rate and column collimation, and weakly on the magnetic field strength. For the UZ For parameters, the observed peak is predicted to be: $\lambda_{\text {peak }} \sim 10 \mu \mathrm{m}\left(1.4 \times 10^{30} / L_{h o t}\right)^{0.5}$. A surprising result is that the cyclotron radiation from a hot phase medium with $L_{\text {hot }} \sim 0.1$ tot could produce significant cyclotron radiation in the near infrared. It should be possible to distinguish the emission from the infalling hot phase material from that created by the underlying shock using broadband eclipse near-IR and thermal-IR data. Hence, we encourage more infrared observations of $\mathrm{mCVs}$ to detect or place strong limits on the hot phase medium in their accretion columns.

Acknowledgments. My collaborators in studies of mCVs are Gary Schmidt and Jim Liebert. The foundation for much of this study was laid by the superb observational and theoretical work of Dayal Wickramasinghe, Lilia Ferrario, Jeremy Bailey, Mark Cropper, and Keith Horne. Support for this work was provided by NASA through grants GO- 2686 from the Space Telescope Science Institute, which is operated by AURA, under NASA contract NAS 5-26555.

\section{References}

Bailey, J. \& Cropper 1991, MNRAS, 253, 27

Beuermann, K. 1988, in "Polarized Radiation of Circumstellar Origin", ed. C. V. Coyne et al. (Vatican City State: Vatican Observatory) 125.

Beuermann, K., Fischer, A., Rousseau. T. \& Woelk, U., 1996, this workshop.

Cropper, M. \& Horne, K. 1994, MNRAS, 267, 481

Dulk, G.A., \& Marsh, K.A. 1982, ApJ, 259, 350

Frank, J., King, A.R., \& Lasota, J.-P. 1988, A\&A, 193, 113

Kuijpers, J. \& Pringle, J.E., 1982, A\&A, 114, L4

Lamb, D.Q. \& Masters, A.R. 1978, ApJ, 234, 117

Paerels, F., Hur, M.Y., Mauche, C.W., \& Heise, J. 1996, ApJ, 464, 884

Ramsay, G., Mason, K.O., Cropper, M., Watson, M.G. \& Clayton, K.L. 1994, MNRAS, 270, 692

Robinson, C.R. \& Cordova, F.A., 1994, ApJ, 437, 436

Stockman, H.S., Schmidt, G.D., Angel, J.R.P, Liebert, J., Tapia, S., \& Beaver, E.A. 1977, ApJ, 217, 815

Stockman, H.S., Schmidt, G.D., Liebert, J., \& Holberg, J.B. 1994, ApJ, 430, 323

Stockman, H. 1995 the Cape Workshop on Magnetic Cataclysmic Variables, ASP Conf. Series, eds. D.A.H. Buckley \& B.Warner, 85, 153

Stockman, H.S., \& Schmidt, G.D. 1996, ApJ, 468, 883

Woelk, U. \& Beuermann, K. 1993, A\&A, 280, 169 


\section{Discussion}

C. Mauche: Regarding the EUV light curve of AM Her, fits to the EUV spectrometer data rule out large variations in the neutral absorbing column with binary phase - the shape of the EUV light curve cannot be explained simply by variations in the absorbing column.

$P$. Stockman: Yes, I had used the wrong terminology, $N_{H}$, in describing the base of the accretion columns. The gas is highly ionised but has significant optical depth in the HeII continuum. Alternatively, if infalling clumps are large and individually opaque, they will form a grey absorber in the average light curve. Further analysis of EUVE and ASCA data should be able to address this issue.

A. King: Just to respond to Chris Mauche's point - I think you would expect the absorption to be "leaky" i.e. highly inhomogeneous. Then the dense blobs act as effectively perfect absorbers and the effect on the spectrum is just a grey shift, rather than showing the spectral signature of a large absorption. 